\title{
BRITISH

\section{PATHOGENESIS OF ECLAMPSIA}

\author{
BY
}

\author{
W. R. ADDIS, M.C., M.B., Ch.B.Ed., F.C.O.G. \\ Honciary Gynaecologist, Salford Royal Hospital ; Honorary Assistant Surgeon for Women, St. Mary's Hospitals, \\ Manchester
}

The sibject of eclampsia has become so overgrown with fact and theory that at times cne as a clinician feels in danger of losing sight of the wood in consideration of the trees. I therefore offer no apology for the following attempt to reduce eclampsia to the form of a diagram.

\section{Acute Glomerulonephritis and Eclampsia}

The resemblance between acute glomerulonephritis and eclampsia in symptoms and morphological change is so close that one might reasonably expect some similarity in pathogenesis in spite of their aetiological difference. In both we find albuminuria, oliguria with a high specific gravity, and absence of retention in the blood of urinary constituents. The morphological change in the kidneys in the two diseases differs in degree rather than in kind. Both are hypertensive conditions, and hypertensive encephalopathy, the characteristic symptom of eclampsia, is also apt to occur in acute nephritis. Both show oedema, and, though in one there is a typical liver lesion absent in the other, this discrepancy, one would suggest, may depend on a difference in aetiology rather than pathogenesis. Although in acute glomerulonephritis, pre-eclampsia, and eclampsia the renal lesion is only one manifestation of a generalized reaction, the kidney is so widely employed, both clinically and in experimental work, as an indicator that one or two generally accepted facts having a special bearing on this subject may be mentioned.

The renal unit, the nephron, consists of Bowman's capsule, the globular upper end of the tubule, and the tubule, comprising a descending limb, ascending limb, and a convoluted portion. The renal arteriole is continued as the vàs afferens. This divides into a number of capillary loops which invaginate the upper surface of the capsule and carry it on their surface, so that the blood is separated from the cavity of the capsule cnly by a single layer of endothelial cells and this layer of flattened epithelium, united by a slight basement membrane. These capillaries reunite on leaving the glomerulus to form the vas efferens. This again divides into a capillary system which, after following the whole course of the tubule, reunites to form a venule. Each nephron has thus its own individual and twofold capillary system.

The capillary pressure in the glomerulus is 100 to 120 $\mathrm{mm}$. Hg. When $40 \mathrm{~mm}$. $\mathrm{Hg}$ has been deducted for the colloid pressure of the plasma after filtration an effective pressure of $80 \mathrm{~mm}$. $\mathrm{Hg}$ is left, as against 5 to $15 \mathrm{~mm} . \mathrm{Hg}$ in the capillaries elsewhere. The glomerulus has no selestive function, acting simply as a filter through which serum with all its soluble contents, except serum globulin and serum albumin, which have too large a molecule to pass through uninjured endothelium, is transferred to the cavity of Bowman's capsule and thence into the tubule.

That the tubule has an active function is shown by the high oxygen consumption of the kidney. Its function is one of selective reabsorption from or concentration of the filtrate ; thus sugar is completely reabsorbed, water according to the need of the body, and salt sufficient to maintain the $p \mathrm{H}$ of the blood. The non-threshold substances such as urea pass with the excess water into the collecting tubules, and so out as urine. For our present purpose, then, the term "renal function" refers solely to the action of the tubule.

\section{The Kidney in Acute Glomerulon:phritis}

Let us take as our starting-point the mcrphological change found in the kidney in acute glomerulonephritis. This is almost entirely confined to the glomerular capillaries and the afferent vas. Apart from an occasional slight cloudy swelling at the proximal end of the tube, the tubules show no change, as one would expect in view of the high specific gravity of the urine (1030) and the absence of retention of urinary constituents in the blocd. Where such a retention might be expected from a fall in the excretion of urea, with a drop in filtration to below an amount sufficient to deal with the total urea production of the body, the fact that an abnormally high blocd urea is not as a rule found is explained by the power of the anasarca fluid to store urea in about the same concentration as that in which it occurs in the blood. This may account for the high death rate in cases of eclampsia without oedema in which, in effect, a uraemic element, however slight, is superimposed.

The essential kidney lesion is dilatation of the afferent vas and glomerular capillaries, with well-marked ischaemia of the tuft. Bell in 1932 demonstrated a hyaline change in the basement membrane to explain the patency of the almost empty capillaries. (Shaw Dunn in 1934 demonstrated by means of Mallory's connective-tissue stain a slight thickening of the basement membrane in the kidney of eclampsia and pre-eclampsia.) The endothelium is thickened and shows a slight tendency to proliferation. The epithelial layer reveals a similar change. A few leucocytes are present. The whole tuft is swollen and completely fills Bowman's capsule, at times showing a tendency to herniate into the tubule. This glomerular change is universal, not focal. The intertubular capillaries are dilated and engorged. 


\section{Pathogenesis of Acute Glomerulonephritis}

There are two main theories as to the pathogenesis of acute glomerulonephritis. The first (which is ascribed by Fishberg to Conheim and Lichtheim, 1877) is based on the fact that the disease occurs in the course of acute infections, especially scarlet fever and tonsillitis. It describes the condition as one of generalized toxic injury to the capillaries, of which the kidney lesion is only one manifestation. This concept of the condition as generalized is supported by the fact that oedema sets in early, simultaneously with or even before the appearance of albuminuria, and by the high protein content of the anasarca fluid. The severity of the capillary change in the glomerulus, through which toxin is eliminated, as compared with that in the intertubular capillaries and those of other organs, was held to indicate its toxic origin.

Against this, however, we find that injection into experimental animals of the toxin or endotoxin of the Streptococcus scarlatinae, though it produces an inflammatory reaction, fails to produce the ischaemia of the tuft characteristic of acute glomerulonephritis. Further, "postscarlatinal glomerulonephritis becomes chronic in only an exceedingly small proportion of cases" (Fishberg). Many cases of chronic nephritis give no history of a previous acute attack, though a history of scarlet fever or of some less acute infection is frequently obtained. As was shown by Trask and Blake in 1924, moreover, the toxin of scarlatina can be demonstrated in the urine, and we know that albuminuria is a frequent symptom in acute infective fevers. It would seem probable that a slight inflammatory change occurs in a proportion of these cases, some of which may later develop chronic nephritis. It does not therefore seem wholly unreasonable to ascribe the inflammatory part of the glomerular lesion in acute glomerulonephritis to the action of the toxin of the scarlet fever, etc., and regard it as in existence before the onset of the nephritis. The essential kidney lesion of acute glomerulonephritis, as found post mortem, one would suggest, is therefore confined to a universal dilatation of the vasa afferentia and capillaries of the tuft with ischaemia.

\section{Volhard's Theory}

In 1923 Volhard put forward another theory of the pathogenesis of acute glomerulonephritis. He suggested that it was the result of a generalized angiospasm producing capillary ischaemia. The endothelium of capillaries accustomed to a pressure of $120 \mathrm{~mm}$. $\mathrm{Hg}$ might reasonably be supposed to be highly susceptible to anoxaemia. It probably undergoes an albuminoid degeneration with the formation of a smaller protein molecule, consequent swelling, and increased permeability. The oliguria may result from decreased flow of blood through the tuft or from a fall in intracapillary pressure. Shaw Dunn has suggested that as the glomerular change is diffuse it is possible that instead of only one in three of the nephrons functioning at one time, as occurs normally, all three are working simultaneously. Each glomerulus in this way filters only a third of the blood it otherwise would. The length of the tubule remaining constant, the filtrate in this circumstance may be regarded as passing over three times the usual length of concentrating epithelium.

Opponents of Volhard's theory ascribe the oliguria to impermeability of the tuft owing to inflammatory swelling. We find, however, that the lumina of the capillaries show a fixed patency and contain a few red blood corpuscles. We also find engorgement of the intertubular capillaries, and, though the presence of some obstruction due to endothelial thickening cannot be denied, Volhard has shown that the kidney can be transfused after death has relieved the arteriole spasm.

\section{The Renal Lesion}

Baird and Shaw Dunn (1933) assessed the position of the renal lesion in eclampsia and pre-eclampsia as lying midway between the normal and that of acute glomerulonephritis. They agree with Bell (1932) that "a distinction can usually easily be made between the two conditions," the distinction being the absence of leucocytes or tendency to cell proliferation in the glomerulus of eclampsia. As this part of the nephritic lesion, one has suggested, is due to the elimination through the tuft of the scarlatinal or other toxin, and as this excretion has as a rule ceased before the onset of the nephritis, we may, I think, for our present purpose justly regard the kidney lesions in the two diseases as identical in kind.

\section{Common Pathogenic Factors}

The main object of this paper is to show that in angiospasm we have a common pathogenic factor underlying and uniting all the varying expressions of eclampsia: Betieving as one does that acute glomerulonephritis is an angiospastic condition, and having indicated an essential morphological similarity between the kidney lesions in the two diseases, let us consider whether the analogy can be carried further to include all the manifestations of both conditions.

The first common symptom is oedema. In both diseases it is of rapid onset. There is a difference in the severity and distribution of the swelling, but in both the high protein content of the anasarca fluid shows it to be due to endothelial injury. There is, however, no clear indication as to the cause of this damage except on an analogy with the other symptoms of the diseases. On the other hand, the pallor of the skin typical of acute nephritis is almost certainly due to angiospasm, and Hinselmann (1924) observed in cases of eclampsia, by means of the capillary microscope, constantly recurring spasmodic contractions of the capillaries at the base of the finger-nail, at times producing complete cessation of flow. Capillary circulation is controlled by the Rouget cells, a layer of unstriped muscle encircling the vessel wall reticulated to allow of transudation of fluid. To these sympathetic fibres can be traced. We know that these cells are absent in the lung and kidney, while in the liver lobule not only are they absent but even the endothelium is incomplete. Further, Williams (1936) has shown that the intracranial capillaries have no sympathetic supply. Accordingly in these organs we must look further back than the capillary for the site of our angiospasm.

In the retina of both diseases a spastic condition of the arterioles can be observed producing indentation of the venules at arterio-venous crossings. In extreme cases oedema can occur of such severity as to cause separation of the retina.

The rise in blood pressure cannot be regarded as a reaction to obstruction to the renal circulation alone, as in acute glomerulonephritis the pressure seldom exceeds $180 \mathrm{~mm}$. $\mathrm{Hg}$, whereas in eclampsia, with a much less severe kidney change, it at times reaches great heights. It is more reasonable to suppose this to be due to a generalized arteriole spasm. In eclampsia with a higher pressure reaction we get a correspondingly wider distribution of lesion referable to angiospasm. 


\section{Hypertensive Encephalopathy}

In this consideration of the manifestations of acute glomerulonephritis and eclampsia as due to angiospasm there is one last common symptom to be taken into account-namely, hypertensive encephalopathy. This term was introduced by Oppenheimer and Fishberg in 1928 to describe the condition which Volhard in 1918 designated "pseudo-uraemia" to distinguish it from uraemia, with which it had previously been confused. Uraemia, as its name implies, is dependent on the presence in the blood of an abnormally high non-protein nitrogen. The condition is insidious in onset, and is preceded by muscular weakness and wasting. Anorexia and diarrhoea occur and the skin is pale and dry, and shows a yellowish-brown discoloration. The urine is as a rule greatly diminished but of low specific gravity (1010), and the urea concentration in the blood is high, indicating failure of renal function. Babinski's sign is absent.

In hypertensive encephalopathy, on the other hand, the onset is dramatic in its suddenness. Vaquez and Nobécourt pointed out in 1897 that the essential feature in eclampsia was arterial hypertension showing a rapid rise just before or coincident with the onset of the seizures. The attacks start with a generalized tonic spasm, which gives way to a clonic convulsion followed by coma. The pupils are dilated and react sluggishly. Babinski's sign can generally be elicited. The pressure of the cerebrospinal fluid is raised, and often greatly increased. No indication of failure of renal function is found on examination of the urine and of the blood.

Hypertensive encephalopathy occurs in three conditions -eclampsia, lead poisoning, and acute glomerulonephritis. All three are hypertensive, and in all the seizures are epileptiform in character. In regard to lead poisoning, Vaquez (1904) showed that the colic occurred with hypertension and was due to constriction of the mesenteric vessels. Similarly the arteriosclerosis of chronic lead poisoning is held to result from spasm of the media. The spastic nature of the condition is further indicated by the pallor of the skin, even in cases where the anaemia is slight, and by the cramps which are a typical feature of the disease.

Post mortem, in these three varieties of encephalopathy the brain is pale and bloodless. Oedema due to capillary ischaemia is often but not invariably present. These findings, considered in conjunction with sudden onset and short duration of the attacks, are very suggestive of an intracranial angiospasm.

Though it has long been realized that the convulsive seizures in eclampsia were in some way associated with cerebral anaemia (as far back as 1859 Kussmaul and Tenner demonstrated epileptiform convulsions following ligation of the arteries to the head in rabbits) the possibility of the phenomenon of hypertensive encephalopathy being caused by cerebral angiospasm was denied by many physiologists on account of the comparative deficiency of the media of the cerebral vessels. In 1932-3 Roberts showed that Cheyne-Stokes breathing produced by adrenaline was accompanied by opening and closing of the cerebral vessels. They considered it due to constriction of the arteries to the respiratory centre. Mellanby and Haggett (1933) confirmed these findings by injecting ergotoxine, one of whose actions is to paralyse the motor side of the sympathetic, and so abolished adrenaline apnoea, indicating that it was due to constriction of the vessels to the bulb. Finally Williams (1936) demonstrated with ample histological evidence the existence of an extensive sympathetic supply to the cerebral vessels with the exception of the capillaries. His final conclusion is as follows: "The sympathetic innervation of the intracranial circulation does not differ materially from that of the systemic circulation." It does not seem reasonable to suppose vessels with a full and normal sympathetic innervation incapable of contraction. If then we accept the possibility of intracranial angiospasm this would seem to be the simplest explanation of the cerebral ischaemia, with or without oedema, which we find post mortem in all three types of hypertensive encephalopathy, more especially as in all three angiospasm can be demonstrated as occurring elsewhere at the same time. Lastly, the presence of Babinski's sign, absent in the irritative seizures of uraemia, indicates a cutting off of cortical control by ischaemia, which it would be difficult to explain by any other mechanism in such a condition as eclampsia.

\section{The Liver Lesion}

Though the liver lesion of eclampsia does not occur in acute glomerulonephritis there would seem to be as much reason to consider it as due to an arteriole spasm with capillary ischaemia as is the glomerular lesion of both diseases. The essential liver lesion in eclampsia is a periportal haemorrhagic necrosis with a similar though naturally less marked change in the intracapsular tissue. The hepatic artery supplies the connective tissue in Glisson's capsule and the sinusoids of the area of lobule surrounding the capsule. It contributes one-quarter of the blood supply to the lobule and 40 per cent. of the oxygen, the main central portion being oxygenated by the portal veins (Starling).

The liver lesion has all the characters of a severe multiple infarction. It is confined to those areas of the lobule supplied by the hepatic arteries-that is, the only areas where angiospasm could produce ischaemia. Barcroft has pointed out the special liability of liver cells to injury from deprivation of oxygen, which explains the severity of the lesion here as compared with the results of angiospastic ischaemia in other organs. In toxic conditions such as acute yellow atrophy the necrosis starts centrally and does not resemble an infarct. Were the eclamptic lesion due to cell injury by some toxic substance circulating in the blood it would be difficult, in view of the open nature of the sinusoids into which the capillaries open, to understand the strict localization of the lesion, in uncomplicated cases, to the periportal areas at the periphery of the lobule. I would then suggest that there is good reason to regard the hepatic lesion in eclampsia as a multiple infarction due to angiospasm of the hepatic arteries or their arterioles.

\section{Other Manifestations of Eclampsia}

There are many manifestations of eclampsia which have not been mentioned-for instance, oedema of the lungs. This I had in mind when the absence of Rouget cells in this situation was mentioned. Ischaemic injury to the alveolar capillaries from intermittent spasm of the pulmonary arterioles would be expected to result in oedema and congestion. This is also accentuated by cardiac failure, the termination of most fatal cases. It has been said that "each fit brings the patient nearer her end." It is true that the number of fits is of great prognostic value. It is not, however, probable that the fits of them- 
selves could lead to a fatal termination: it is rather that the seizure is the outward and visible sign of a sudden access of angiospasm, which must affect the myocardium just as it affects practically all the tissues of the body.

Lastly, there is one other symptom of grave prognostic value which deserves mention-namely, a rise in temperature. This may in part be due to decreased loss of heat during the exertion of the seizures by spasm of the superficial capillaries. This alone would, however, be a completely inadequate explanation. Pyrexia, one would suggest, is the expression of an exceptionally severe angiospasm. In anaesthesia the first things to go are consciousness and the power of voluntary movement. As the anaesthesia advances it spreads downwards and gradually affects the lower centres in the pons and medulla, ultimately causing death. So with ischaemia, a moderate degree will knock out the higher, cerebral, portion of the brain while the lower centres remain comparatively unaffected. Only a very severe angiospasm will suffice to affect the heat-regulating centre in the corpus striatum. It is not surprising that the prognosis in surch a case is grave.

\section{Commentary}

It would be impossible in the space of a short paper to cover the infinite variations of such a disease as eclampsia. All one can hope to do is to include what are considered to be essential features alone. I have merely attempted to draw a diagram. Such a diagram I conceive as having at its centre angiospasm. This spreads out into various tissues and organs, producing effects which in themselves appear to be completely unrelated. In the brain it results in hypertensive encephalopathy; in the kidney, albuminuria and oliguria with a high specific gravity; in the liver, a periportal necrosis; in the subcutaneous tissues, anasarca; and in the skin, though it is frequently masked by deficient oxygenation of the blood, pallor. I would suggest that these variations are the result not of a varying cause but of the variety of tissues in which it acts, and that there is one single pathogenic factor underlying and uniting all the manifestations of eclampsianamely, angiospasm.

The fourth annual report of the Research Institute and the Endemic Diseases Hospital, published by the Ministry of Public Health, Egypt, deals with the year 1934. During this period an investigation of the chemical composition of the spleen in Egyptian splenomegaly was initiated, and the results of analysis will be given in a subsequent report. The urine of 116 adult females attending child welfare centres in Cairo was examined for bilharzial infection, which was discovered in forty-four cases. Nine of these women had never left Cairo. Further inquiry showed that eight of the nine lived in suburban districts, where filtered water was not available. Bulimus snails were discovered in the streams from which these women drew their water supply, although no cercariae were to be found. Schistosoma infection was very high in those parts of the country where systems of continuous irrigation have been introduced, the percentage reaching 75 in some districts. In the clinical section of the report it is stated that fouadin is used for the routine treatment of schistosomiasis. Examination of the urine of 11,635 out-patients demonstrated the presence of ova of Schistosoma haematobium in 45.7 per cent. ; and stools contained the ova of Ankylostoma duodenale, Ascaris lumbricoides, and Trichostrongylus in 28.2, 16.8, and 12.8 per cent. of cases respectively. Ankylostomiasis is treated by carbon tetrachloride with satisfactory results.

\section{INTERMITTENT VENOUS OCCLUSION IN THE TREATMENT OF OBLITERATIVE VASCULAR DISEASE \\ BY}

\author{
J. J. MASON BROWN, M.B., F.R.C.S.Ed. \\ Assistant Surgeon, Royal Hospital for Sick Children, \\ Edinburgh \\ AND
}

W. MELVILLE ARNOTT, M.B., B.Sc. M.R.C.P.Ed., Lecturer in Therapeutics, University of Edinburgh (From the Laboratories of Clinical Medicine and Clinical Surgery, Royal Infirmary, Edinburgh)

Obliterative vascular disease, with its inevitable sequelae of intolerable pain and progressive gangrene, provides one of the most baffling and melancholy problems in both medical and surgical practice. It is especially tragic in the young or middle-aged patient with thrombo-angiitis obliterans in whom amputation of one or more limbs is a common event. It is clear, therefore, that any method of treatment which holds out a prospect of improvement in the peripheral circulation merits attention. Division of the sympathetic nerve supply in such cases can only increase the blood supply in so far as the obstruction is due to associated vasospasm. In those cases in which obliterative organic disease is the dominant factor in the obstruction it is obvious that some other line of therapy must be employed.

Lewis and Grant (1925) in a study of reactive hyperaemia in man found that when the arterial supply to a limb was occluded there resulted a state of vasodilatation lasting from half to three-quarters of the period of circulatory arrest. This dilatation involved both the superficial and deep vessels of the limb and was independent of the central nervous system and local reflexes. An exactly comparable reaction followed increase of venous pressure in a limb. It was apparent at as low a cuff pressure as $40 \mathrm{~mm} . \mathrm{Hg}$, and became more pronounced up to the point of occlusion of arterial flow. They concluded that the vasodilatation affected the capillaries and smaller arterioles only and was caused by the accumulation in the extravascular tissue fluids of slowly diffusible substances produced during the period of circulatory disturbance. Very recently Barsoum and Smirk (1936) have studied venous blood from limbs in a state of reactive hyperaemia. They found an increase in the concentration of a substance which resembled histamine in its biological properties.

\section{The Apparatus}

From a consideration of Lewis and Grant's work Collens and Wilensky (1936a) devised an apparatus which raised the pressure in a cuff encircling the limb to any desired pressure in cycles of two minutes' pressure followed by two minutes' release, and in a more recent paper (1936b) have reported the result of the treatment of a small series of cases of obliterative vascular disease by this method. The results were strikingly successful, pain being almost immediately relieved, ulcers healed, and gangrene averted. We have had an apparatus constructed for this purpose, and it has now been in use since the beginning of February. We had intended to observe the results of this treatment in a large series of cases before publication, but the editorial appeal in the March 6 issue of the British Medical Journal for the production of a simple apparatus for this form of therapy indicated that a description of our apparatus might be of interest. It is simpler in construction than that devised by Collens and Wilensky. 